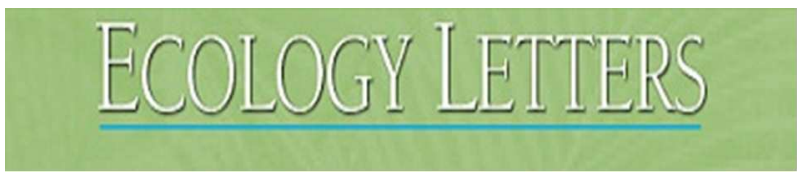

\title{
Community fluctuations and local extinction in a planktonic food web
}

\begin{tabular}{|r|l|}
\hline Journal: & Ecology Letters \\
\hline Manuscript ID & Draft \\
\hline Manuscript Type: & Letters \\
\hline Date Submitted by the Author: & n/a \\
\hline Complete List of Authors: & $\begin{array}{l}\text { Segura, Angel; Universidad de la Republica Uruguay - Centro Universitario } \\
\text { de la Region Este - Sede Rocha, Rocha } \\
\text { Calliari, Danilo; Universidad de la Republica, Facultad de Ciencias, } \\
\text { Ecologiia, Seccion Oceanografiia } \\
\text { Lan, Leong; Monash University, School of Science } \\
\text { Fort, Hugo; Universidad de la República, Institute of Physics } \\
\text { Widdicombe, Claire; PML, MLSS } \\
\text { Harmer, Rachel; PML, MLSS } \\
\text { Arim, Matias; Centro Universitario Regional Este (CURE), Universidad de la } \\
\text { República, Ecología Teórica y Aplicada; Facultad de Ciencias, universidad } \\
\text { de la República, Ecología y Evolución }\end{array}$ \\
\hline Key Words: & $\begin{array}{l}\text { macroecology, plankton, food web, fluctuations, Station L4, English } \\
\text { channel }\end{array}$ \\
\hline \hline
\end{tabular}


1 Community fluctuations and local extinction in a planktonic food web

3 Authors: Segura, A.M. ${ }^{a}$, Calliari, D. ${ }^{a}$, Lan, B.L., ${ }^{\text {bc }}$, Fort, H. ${ }^{\mathrm{b}}$, Widdicombe, C.E. ${ }^{\mathrm{d}}$ Harmer, R. ${ }^{\mathrm{d}, \mathrm{e}}$, Arím, $4 M^{\mathrm{a}}$

5 Running title: Community stability and local extinction Affiliations

$6 \quad{ }^{a}$ Universidad de la República, Centro Universitario Regional Este

7 b Universidad de la República, Physics Institute, Complex systems group, Montevideo, Uruguay

8 c Electrical and Computer Systems Engineering, School of Engineering, Monash University, 47500 Bandar Sunway, 9 Malaysia

10 d Plymouth Marine Laboratory, Prospect Place, West Hoe, Plymouth PL1 3DH, United Kingdom

11 e Institut für Hydrobiologie und Fischereiwissenschaft, Universität Hamburg, Hamburg, Germany

Corresponding author:

Angel M. Segura

Centro Universitario Región Este, Ruta 9 y 15, P.C. 27000, Rocha, Uruguay

e-mail: asegura@,cure.edu.uy

Type: Letter

\# of words: 3023 in abstract: 251

\# Figures: 4

\# Citations: 41

Authorship: A.M.S. Designed and performed analysis and wrote a first draft. H.F. and L.B. Contributed with analytical tools. C.W. and R.H. generated the database. M.A. and D.C. and L.B. discussed results. All authors contributed to the writing of the paper. 


\section{Data accessibility statement:}

Station L4 data are archived at the British Oceanographic data centre www.bodc.ac.uk and are freely available upon request to Dr Angus Atkinson aat@pml.ac.uk

10

12

13

14

15

16

17

18

19

20

21

22

23

24

25

26

27

28

29

30

31

32

33

34

35

36

37

38

39

40

41

42

43

44

45

46

47

48

49

50

51

52

53

54

55

56

57

58

59

60 
12 Abstract

13 Determining statistical patterns irrespective of interacting agents (i.e. macroecology) is useful to 14 explore the mechanisms driving population fluctuations and extinctions in natural food webs. Here, we 15 tested four predictions of a neutral model on the distribution of community fluctuations (CF) and the 16 distributions of persistence times (APT). Novel predictions for the food web were generated by 17 combining i) body size-density scaling, ii) Taylor's law and iii) low efficiency of trophic transference. 18 Predictions were evaluated on Fan exceptional dataset of plankton with 15 years of weekly samples 19 encompassing $\sim 250$ planktonic species from three trophic levels, sampled in the western English 20 Channel. Highly symmetric non-Gaussian distributions of CF supports zero-sum dynamics. Variability 21 of CF decreased while a change from an exponential to a power-law distribution of APT from basal to 22 upper trophic positions was detected. Results suggest a predictable but profound effect of trophic 23 position on fluctuations and extinction in natural communities.

25 Key words: macroecology, plankton, food web, fluctuations, Station L4, English channel 26 27 


\section{INTRODUCTION}

29 The macroecological perspective represents a fruitful and complementary approach to 30 traditional methods in ecology which may provide novel insights on patterns and process shaping 31 biodiversity (John Maynard Smith 1974; Maurer 1999; Harte 2011; Hatton et al. 2015). Such a 32 macroscopic approach could be particularly suitable for the analysis of community dynamics involving 33 hundreds of interacting species. The aggregation of population fluctuations $r_{s}=\log _{e}\left(N_{t+1} / N_{t}\right)$ of multiple 34 species results in a distribution of community fluctuations (CF) that departs from Gaussian but follows 35 a Laplace probability distribution (Keitt \& Stanley 1998):

36

$37 P\left(r_{s}\right)=\frac{1}{(2 \Phi)} e^{\frac{-\left|r_{s}-\mu\right|}{\Phi}}$

(eq. 1)

38

39 where $\mu$ is the mean and $2 \Phi^{2}$ is the variance $\left(\sigma_{\mathrm{r}}^{2}\right)$. The distribution extended several orders of 40 magnitude, was symmetric and centered on zero suggesting that demographic gains and losses by all 41 the species were balanced over the study period (Marquet et al. 2007). That analysis combined species 42 spanning a wide range of body-sizes feeding at multiple trophic levels. Consistent relationship of 43 trophic position with body size (Arim 2007; Segura et al. 2015), and the association of body size with 44 decreased growth rate (Brown et al. 2004) increased scale of perception (Ritchie 2010; Borthagaray et 45 al. 2012) and coupling of energy channels (Rooney et al. 2006; Arim et al. 2010; Rooney \& McCann 46 2012) are expected to drive systematic trends in population fluctuation and trophic position. Moreover, 47 while the propagation of community fluctuations through the food web has been a cornerstone of 48 community ecology (Stouffer \& Bascompte 2011; Thompson et al. 2012) its connection with the 49 distribution of populations' fluctuation has not been considered so far. 
50 The distribution of species aggregated persistence time (APT) has been proposed as a novel 51 macroecological pattern that reflects important ecological processes (Bertuzzo et al. 2011; Suweis et 52 al. 2012). Persistence time of a species was defined as the time span between local colonization and 53 extinction in a given geographic region. APT showed a power-law scaling with exponential cut-off for 54 bird communities and a qualitative relationship between CF and APT was proposed, but no formal link 55 was suggested (Keitt \& Stanley 1998). Recently, a formal connection between APT and CF was 56 proposed for neutral systems (Pigolotti et al. 2005; Bertuzzo et al. 2011). Under neutral dynamics, it 57 was demonstrated that APT distribution followed a power law with an exponential cut-off of the form 58 (Bertuzzo et al. 2011):

$59 \mathrm{P}(\mathrm{t}) \sim \mathrm{t}^{-\alpha} \mathrm{e}^{-v \mathrm{t}}$ (eq. 2)

60 When dispersal is unlimited, the scaling exponent $(\alpha)$ equals 2 and $\mathrm{P}(\mathrm{t})$ depends solely on the 61 immigration or speciation rate $(v)$. Ecosystem dimensionality and spatial constraints on dispersal 62 modify the scaling exponent in the range $\alpha=1.5-2$ (Pigolotti et al. 2005; Bertuzzo et al. 2011) as was 63 observed for breeding birds, herbaceous plants and marine fishes (Bertuzzo et al. 2011; Suweis et al. 64 2012). Although the supporting evidence is compelling, the neutral model is limited to explain patterns 65 in trophically equivalent neutral species.

66 A way towards advancing our understanding of food web dynamics relies on the evaluation of 67 hypotheses under the macroscopic lens. First, the integration of multiple energetic pathways by 68 predators, was shown to be a powerful mechanism to stabilize predator dynamic and the whole food 69 web (Rooney et al. 2006; Rooney \& McCann 2012). Second, the negative scaling of body size and 70 population variance (i.e. variance mass allometry, VMA) predicts a smaller variance in large-sized 71 species (Cohen et al. 2012). Assuming a size structured food web, and low efficiency of energy 
72 transference between trophic levels, a relatively lower population variance in predators with respect to 73 preys is expected. Both mechanisms, the coupling of multiple energy channels and the negative scaling

74 of variance with body size are not mutually exclusive and predict a systematic decrease in community 75 fluctuations and colonization and extinction at higher trophic levels. A main limitation for the 76 evaluation of these predictions in particular and the empirical analysis of fluctuations and persistence 77 distribution in general, is the lack of appropriate information. A proper representation of both 78 persistence times and fluctuations require data for multiple species during long time periods, typically 79 several generations, which is seldom available, but plankton is the exception.

80 The base of oceanic food webs is composed of microscopic unicellular producers (e.g. diatoms)

81 which are consumed by a complex array of predators including unicellular protists (e.g. cilliates) and 82 crustacean metazoans (e.g. copepods) (Segura et al. 2013). Plankton dynamics regulate the flux of 83 matter and energy towards upper trophic levels that in turn support fisheries and the exchange of $\mathrm{CO} 2$ 84 with the atmosphere. Empirical evidence suggests that planktonic predators integrate different energetic 85 pathways (Rooney et al. 2008), show similarities in scaling relationships to terrestrial organisms 86 (DeLong \& Vasseur 2012; Hatton et al. 2015) and present a wide range of dispersal strategies. Here, 87 using the western English Channel Station L4 data composed of fifteen years of weekly information 88 ( $\sim 800$ weeks) on abundance and size of more than 250 planktonic species, we tested the validity of the 89 following predictions of the Neutral theory: 1) the distribution of community fluctuations (CF) at 90 different trophic levels conformed to a symmetric distribution, 2) community fluctuations variance 91 decreased with trophic level, 3) the aggregated persistence times distribution (APT) follows a truncated 92 power law distribution with scaling exponent between 1.5 and 2.0, and 4) the existence of a negative 93 relationship between CF and APT. 


\section{MATERIAL and METHODS}

95 Sampling and species counting

96 Sampling for the planktonic community (phyto-, microzoo- and zooplankton) at Station L4 in 97 the western English Channel was conducted weekly from 1988 (Eloire et al. 2010; Widdicombe et al. 98 2010). However a gap in phytoplankton sampling between 1994 and 1995 meant that we started our 99 series analysis with data from 1995 to 2012 ( $~ 800$ weeks). Species were grouped in three coarse 100 trophic groups i) primary producers, ii) consumers and iii) predators. Primary producers (diatoms and 101 dinoflagellates) and consumers (ciliates and heterotrophic dinoflagellates) were identified and 102 enumerated using settlement microscopy (Utermöhl, 1958) while predators (i.e. copepods) were 103 identified and counted using a dissecting microscope. For a detailed description of sampling and 104 counting methods we refer to (Widdicombe et al. 2010) for primary producers and consumers and 105 (Eloire et al. 2010) for predators. Diatoms and dinoflagellates are microbial producers ranging from 5 106 to $100 \mu \mathrm{m}$ in spherical equivalent diameter (ESD), while ciliates and heterotrophic dinoflagellates are 107 mostly heterotrophic unicellular predators ranging from 20 to $200 \mu \mathrm{m}$ ESD. It is worth to mention that 108 most of these organisms are mixotrophic. Copepods are multi-cellular crustaceans with complex life 109 cycles and size ranging from 200 to $1000 \mu \mathrm{m}$ ESD. As copepods present several feeding modes 110 (Hansen et al. 1994) they can be classified in several trophic levels. For copepods, we performed the 


\section{Data analysis}

118 Population fluctuations $\left(\mathrm{r}_{\mathrm{s}}\right)$ were estimated for each species by dividing abundance $\left(\mathrm{N}_{\mathrm{t}+1}\right)$ by 119 abundance in the preceding week $\left(\mathrm{N}_{\mathrm{t}}\right)$ and taking the logarithm of the ratio as in (Segura et al. 2013):

$121 \mathrm{r}_{\mathrm{s}}=\log \left(\mathrm{N}_{\mathrm{t}+1} / \mathrm{N}_{\mathrm{t}}\right)$

Next, we aggregated the population fluctuations of all species belonging to the same functional group into a single vector. These aggregated population fluctuations or community fluctuations (CF) were 125 fitted with a Laplace distribution (eq. 1) where the location $(\mu)$ and scale $(\Phi>0)$ were estimated with 126 the VGAM package (Yee 2010) in statistical software R (R Core Team 2013). We evaluated if the 127 distribution conformed to a Laplace by means of the Kolmogorov-Smirnov test. In order to evaluate 128 zero sum dynamics, we tested if zero was included in the $95 \%$ confidence interval of $\mu$. We evaluated 129 symmetry around the median of the CF distribution with the Miao, Gel and Gastwirth (MGG) test 130 (Miao et al. 2006) as implemented in the package lawstat (Gastwirth et al. 2015).

In order to estimate persistence time, we interpolated linearly for each species the abundance 132 time series as some samplings were not performed exactly every seven days. For each species, we 
1
2
3
4

5139 evaluated the power law distribution with exponential cut-off excluding the probability of observing a 6

7140 persistence of 1 week.

8

$9 \begin{array}{ll}9 & 141\end{array}$

11

12

13

14

15

16

17

18

19

20

21

22

23

24

25

26

27

28

29

30

31

32

33

34

35

36

37

38

39

40

41

42

43

44

45

46

47

48

49

50

51

52

53

54

55

56

57

58

59

60 


\section{RESULTS}

A total of 38695 abundances were recorded in the study period. Community fluctuations were 144 calculated for 253 species classified in five phylogenetic groups including 128 Diatoms, 38

145 Dinoflagellates, 30 Ciliates, 38 Heterotrophic Dinoflagellates and 35 Copepod species. They were 146 aggregated into three functional groups: primary producers, consumers and predators. CF in all 147 functional groups were long-tailed and visually conformed to a Laplace distribution (Fig. 1) although 148 formal tests rejected this hypothesis (K-S; D $>2 ; \mathrm{p}>0.05$ ). Mean over all groups was not different from 149 zero (average (s.d.) $=0.001(0.006))$ and the distributions were symmetric around the median (MGG 150 test, $\mathrm{p}>0.05$ ) for all groups except for the heterotrophic dinoflagellates (MGG test, $\mathrm{p}<0.01$ ). As 151 expected in our working hypothesis, we found a consistent trend in community variability decreasing 152 from primary producers (Diatoms $\sigma_{\mathrm{r}}^{2}=2.89$; Dinoflagellates $\sigma_{\mathrm{r}}^{2}=2.57$ ), to consumers (Ciliates $\sigma_{\mathrm{r}}^{2}=$ 153 1.78; Heterotrophic dinoflagellates $\sigma_{\mathrm{r}}^{2}=1.45$ ), to predators (Copepods $\sigma_{\mathrm{r}}^{2}=1.39$ and Omnivorous 154 copepods $\left.\sigma_{\mathrm{r}}^{2}=1.25\right)$.

155 A power-law distribution with an exponential cut-off, Eq. 2, adequately described aggregated 156 persistence times (APT) for all functional groups (Fig. 2). The APT scaling exponent ( $\alpha$ ) systematically 157 increased across trophic levels and ranged from 0.58 to 1.19 , values that are lower than predictions 158 based on neutral models (Bertuzzo et al. 2011). We found a strong negative correlation of the APT 159 scaling exponent $(\alpha)$ with the exponential decay rate $(v)$ (Pearson's $r=-0.98 ; P<0.01 ; \mathrm{N}=6$ ). 160 Consequently, the shape of the persistence times distribution shifted from an exponential regime at 161 lower trophic levels towards a power law regime at higher trophic levels, and the characteristic 162 persistence time ( $\tau=1 / v)$ increased systematically from producers to predators (Fig. 3). 
1

2

3

4

5

6

7

8

9 10 11 12 13

164 (v) (Pearson's $r=0.92, p<0.01 ; \mathrm{N}=6$ ). This implies that populations at lower trophic levels tend to be 165 less persistent and more fluctuating than those at higher trophic levels (Fig. 3). Average group's body166 size partially explained the difference in the variability and persistence between large-sized copepods 167 and unicellular species. However, as predicted by our working hypothesis, trophic level alone also 168 explained a significant fraction of the differences in fluctuations and persistence time distributions 169 among similar-sized species (e.g. dinoflagellates, ciliates and heterotrophic dinoflagellates; Fig. 4). 


\section{DISCUSSION}

171 We showed a consistent increase in community stability (decreased variability) and persistence times

172 towards higher trophic levels in the species rich planktonic food web of the western English Channel.

173 Three remarkable patterns were documented among trophic levels, i) the symmetry in populations

174 fluctuations, ii) the decrease in variance of community fluctuations with body size and trophic level and

175 iii) the systematic shift in the shape of the persistence times distribution with increasing trophic level.

176 Consequently, the macroecological distributions herein analyzed provides a complementary support for 177 current food web hypotheses (Hubbell 2001; Otto et al. 2007; Bertuzzo et al. 2011; McCann 2012).

As expected, we found a systematic decrease in the variance of community fluctuations with 179 body size and increasing trophic level (Cohen et al. 2012). Such patterns can be explained in terms of 180 three main ecological principles: i) the Taylor law, which asserts that the variance of the population 181 density of a set of populations is a power-law function of the mean population density (Taylor 1961), ii) 182 the body size-density power law, according to which the mean population density of a set of 183 populations is a negative power law of the organism body size (Peters \& Wassenberg 1983; Kruk et al. 184 2010) and iii) the fraction of energy lost at each trophic transference (Lindeman 1942) following the 185 second law of thermodynamics. The former two were combined recently by Cohen et al., (Cohen et al. 186 2012) who coined the term variance-mass allometry (VMA) predicting a negative scaling of variance 187 with body size within a single trophic level. Cohen et al., (Cohen et al. 2012) stated that "if VMA [is] 188 applied to marine or freshwater food webs, population densities of smaller-bodied species should be 
193 \& 2). This result expands the VMA for multiple trophic levels accounting for the fact that for similarly 194 sized species, an increase in trophic level implies a decrease in average abundance (Widdicombe et al. 195 2010) and a reduction in the populations fluctuations and an increase in persistence. The progressive 196 addition of energy channels with increasing body size represent a plausible mechanism to drive this 197 pattern (Rooney et al. 2006; Arim et al. 2010; McCann 2012).

198 The negative relationship between persistence times of populations and community fluctuations 199 supports previous predictions (Keitt \& Stanley 1998; Pigolotti et al. 2005; Bertuzzo et al. 2011). It is 20

intuitive that species with large fluctuations will face higher extinction risks, but the specific shape of the distribution of persistence times is also significant. Unexpectedly, we found that the shape of persistence time distribution varied systematically with trophic position (Fig. 3). The evidence of zerosum dynamics at all levels together with the decrease of characteristic persistence timescale ( $\tau$; Fig. 3 ) towards producers suggests that the recruitment and extinction rates are higher at the base and decreases towards the top of the food web as expected (Bertuzzo et al. 2011). However, the slope of the power law $(\alpha)$ was significantly shallower than expected by any of the predictions of the neutral model, irrespective of the structure of the spatial interaction network (Bertuzzo et al. 2011) This point to a profound effect of trophic dynamics in the shape of the persistence times distribution. Such changes in the shape of the distribution had not been described previously because analyses had been conducted either pooling occurrence data from trophically heterogeneous species (Keitt \& Stanley 1998; Bertuzzo et al. 2011) or were based on single trophic level populations data from different systems (Suweis et al. 212 2012). It was suggested that the exponential term could be a statistical artifact caused by the short 213 window of observation of the phenomenon (Keitt \& Stanley 1998; Bertuzzo et al. 2011). Current 214 results based on 200 (copepods) to 500 generations (primary producers) indicate otherwise. Also, the 
215 exponential term $(v)$ decreased with trophic position where most persistent groups showed the 216 minimum value. The systematic change in the persistence time distribution with trophic position 217 deserves further scrutiny both in theoretical and empirical grounds.

The prevalence of symmetric and long tailed distributions of population fluctuations across trophic levels and phylogentic groups reported here points to a universal set of driving processes (Fig. 1). These distributions further support the prevalence of balancing processes (i.e. zero-sum dynamics), in which a decline in one population is offset by an increase in other, at least at the large temporal scale of present observations (Hubbell 2001; Labra et al. 2008). This pattern is general within and among trophic levels and seems to be independent of species richness. Previous time series analyses determined that compensatory dynamics, a special case of balancing processes, were common in the plankton, but synchrony (i.e., non-compensatory) was also recorded at specific scales of analyses (Vasseur \& Gaedke 2007). The use of community fluctuations describe balancing processes without specifying the mechanisms (e.g. compensation, statistical averaging). However, community 228 fluctuations represents a complete description of the concept of asynchrony in resource populations 229 fluctuations (e.g. slow vs fast channels). The simplifying dichotomy of slow and fast channels 230 involving asynchronous dynamics is expanded here to the whole food web, and evidenced by the 231 observed compensatory dynamics, a prerequisite for food web stability (Rooney et al. 2006).

Distributions of fluctuations failed a formal test of Laplace, a fact previously mentioned (Keitt $233 \&$ Stanley 1998) and recently evaluated (Kalyuzhny et al. 2014). In spite of formal test rejection, given 234 the relatively good visual fit (Fig. 1) and parsimony principle, it is suggested to keep the Laplace 235 distribution as a good statistical model to explore community fluctuations at large scales. A plausible 236 explanation of the failure relies on the fact that Laplace is the result of mixing random iterates from 
237 normal distributions whose variances follow an exponential distribution (Kotz et al. 2001). If variances 238 of constituent distribution are not distributed exactly as an exponential distribution or constituent 239 distributions are fat tailed (Segura et al. 2013), a mixture between Gaussian and Lapace distribution is 240 expected as it seems to be the case here. In this sense, we caution the use of Central Limit Theorem to anticipate a Gaussian distribution as the default null model. Indeed, the deviations from Gaussian expectations can give insight into the driving ecological mechanisms. An exponential distribution of variances of population fluctuations implies the existence of many populations with reduced variability and a few populations with large fluctuations. This is congruent with the existence of fast and slow energy channels in plankton dynamics (Rooney et al. 2006, 2008; McCann 2012). Populations within the fast channel are expected to show large fluctuation in opposition to the expected dynamics on the slow channel, with an overall strong role on the stabilization of food webs (Rooney \& McCann 2012). The empirical data should encompass the scale at which hypothetical mechanisms are expected to operate (Levin 1992). The analysis of statistical distribution of populations' dynamics, represented in fluctuations and persistence times, requires a large set of populations and a time span of several generations (Keitt \& Stanley 1998). The database of plankton in the western English Channel notably fulfills these requirements. Congruently, clear distributions of populations' fluctuations and persistence times were observed. Further, it was possible to relate the structure of these distributions with the body size and trophic position of the functional groups considered. More importantly, the observed patterns provide complementary support for sound theories previously analyzed with other approaches. That is 256 the case for the variance-mass scaling of Cohen et al., (Cohen et al. 2012) and the stabilization of 257 populations and food web dynamics through the integration of asynchronous energy channels of 258 Rooney et al., (Rooney et al. 2006). Further, novel trends are suggested as the change from an 
259 exponential to a power-law distribution of persistence time from basal to upper trophic positions which 260 should be further explored. The framework provides explicit evaluation of community dynamics and 261 brings novel explicit predictions by the integration of divergent hypotheses that should be evaluated 262 theoretically and empirically.

263

264 Acknowledgment

265 This research was funded by a grant to AMS (ANII-FCE_3_2013_1_100394).

20

21

22

23

24

25

26

27

28

29

30

31

32

33

34

35

36

37

38

39

40

41

42

43

44

45

46

47

48

49

50

51

52

53

54

55

56

57

58

59

60 


\section{REFERENCES}

1.

Arim, M., Abades, S., Laufer, G., Loureiro, M. \& Marquet, P.A. (2010). Food web structure and body size: trophic position and resource acquisition. Oikos, 119, 147-153.

2.

Arim, M., Bozinovic, F. \& Marquet, A. (2007). On the relationship between trophic position, body mass and temperature: reformulating the energy limitation hypothesis. Oikos, 116, 1524-1530.

3.

Bertuzzo, E., Suweis, S., Mari, L., Maritan, A., Rodríguez-Iturbe, I. \& Rinaldo, A. (2011). Spatial effects on species persistence and implications for biodiversity. Proceedings of the National Academy of Sciences, 108, 4346-4351.

4.

Borthagaray, A.I., Arim, M. \& Marquet, P.A. (2012). Connecting landscape structure and patterns in body size distributions. Oikos, 121, 697-710.

5.

Brown, J.H., Gillooly, J.F., Allen, A.P., Savage, V.M. \& West, G.B. (2004). Toward a metabolic theory of ecology. Ecology, 85, 1771- 1789.

6.

Cohen, J.E., Xu, M. \& Schuster, W.S.F. (2012). Allometric scaling of population variance with mean body size is predicted from Taylor's law and density-mass allometry. Proceedings of the National Academy of Sciences, 109, 15829- 15834.

7.

DeLong, J.P. \& Vasseur, D.A. (2012). Size-density scaling in protists and the links between consumerresource interaction parameters. Journal of Animal Ecology, 81, 1193-1201.

8.

Eloire, D., Somerfield, P.J., Conway, D.V.P., Halsband-Lenk, C., Harris, R. \& Bonnet, D. (2010). Temporal variability and community composition of zooplankton at station L4 in the Western Channel: 20 years of sampling. Journal of plankton research, 32, $657-679$.

9.

Gastwirth, J.L., Gel, J.R., Wallace, W.L., Miao, W. \& Noguchi, K. (2015). lawstat: Tools for Biostatistics, Public Policy, and Law. $R$ package version 2.5. http://CRAN.Rproject.org/package=lawstat. 
10.

Hansen, B., Bjorsen, P.K. \& Hansen, P.J. (1994). The size ratio between planktonic predators and their prey. Limnology and Oceanography, 39, 395-403.

11.

Harte, J. (2011). Maximum entropy and ecology: a theory of abundance, distribution, and energetics. Oxford series in ecology and evolution. Oxford University Press, Oxford ; New York.

12.

Hatton, I.A., McCann, K.S., Fryxell, J.M., Davies, T.J., Smerlak, M., Sinclair, A.R.E., et al. (2015). The predator-prey power law: Biomass scaling across terrestrial and aquatic biomes. Science, 349, aac6284aac6284.

13.

Hubbell, S.P. (2001). The Unified Neutral Theory of Biodiversity and Biogeography. Princeton University Press, Princeton.

14.

John Maynard Smith. (1974). Models in ecology. University Press, Cambridge [Eng.].

15.

Kalyuzhny, M., Seri, E., Chocron, R., Flather, C.H., Kadmon, R. \& Shnerb, N.M. (2014). Niche versus Neutrality: A Dynamical Analysis. The American Naturalist, 184, 439-446.

16.

Keitt, H.T. \& Stanley, H.E. (1998). Dynamics of North American breeding bird populations. Nature, 393, 257-260.

17.

Kotz, S., Kozubowski, T.J. \& Podgórski, K. (2001). The Laplace Distribution and Generalizations. A Revisit with Applications to Communications, Economics, Engineering, and Finance.

18.

Kruk, C., Huszar, V.L.M., Peeters, E.T.H.M., Bonilla, S., Costa, L., LüRling, M., et al. (2010). A morphological classification capturing functional variation in phytoplankton. Freshwater Biology, 55, 614-627.

19.

Labra, F., Marquet, P.A. \& Bozinovic, F. (2008). Scaling metabolic rate fluctuations. Proccedings of the National Academy of Sciences of the United States of America, 104, 10900-10903.

20.

Levin, S.A. (1992). The problem of pattern and scale in ecology. Ecology, 73, 1943:1967. 
21.

Lindeman, R.L. (1942). The Trophic-Dynamic Aspect of Ecology. Ecology, 23, 399-417.

22.

Marquet, P.A., Abades, S.R. \& Labra, F.A. (2007). Biodiversity power laws. In: Scaling Biodiversity (ed. D. Storch, P.A.M.J.H.B.). Cambridge University Press, Cambridge., pp. 441-461.

23.

Maurer, B.A. (1999). Untangling ecological complexity: the macroscopic perspective. University of Chicago Press, Chicago, Ill.

24.

McCann, K.S. (2012). Food Webs. Princeton University Press, Oxford and Princeton.

25.

Miao, W., Gel, Y.R. \& Gastwirth, J.L. (2006). A New Test of Symmetry about an Unknown Median. In: Random Walk, Sequential Analysis and Related Topics - A Festschrift in Honor of Yuan-Shih Chow (ed. Agnes Hsiung, C.-H.Z., and Zhiliang Ying). World Scientific Publisher, Singapore., Singapore.

26.

Otto, S.B., Rall, B.C. \& Brose, U. (2007). Allometric degree distributions facilitate food-web stability. Nature, 450, 1226-1229.

27.

Peters, R.H. \& Wassenberg, K. (1983). The effect of body size on animal abundance. Oecologia, 6, 6089.

28.

Pigolotti, S., Flammini, A., Marsili, M. \& Maritan, A. (2005). Species lifetime distribution for simple models of ecologies. Proccedings of the National Academy of Sciences of the United States of America, $102,15747-15751$.

29.

R Core Team. (2013). R: a language and environment for statistical computing. Vienna: R Foundation for Statistical Computing; Vienna, Austria.

30.

Ritchie, M.E. (2010). Scale, heteogeneity, and the structure and diveristy of ecological communities. Princeton University Press, Princeton.

31.

Rooney, N. \& McCann, K.S. (2012). Integrating food web diversity, structure and stability. Trends in 
Ecology \& Evolution, 27, 40-46.

32.

Rooney, N., McCann, K.S., Gellner, G. \& Moore, J.C. (2006). Structural asymmetry and the stability of diverse food webs. Nature, 442, 265-269.

33.

Rooney, N., McCann, K.S. \& Moore, J.C. (2008). A landscape theory for food web architecture. Ecology letters, 11, 867-881.

34.

Segura, A.M., Calliari, D., Fort, H. \& Lan, B.L. (2013). Fat tails in marine microbial population fluctuations. Oikos, 122, 1739-1745.

35.

Segura, A.M., Franco-Trecu, V., Franco-Fraguas, P., Arim, M. \& Tonn, W. (2015). Gape and energy limitation determine a humped relationship between trophic position and body size. Canadian Journal of Fisheries and Aquatic Sciences, 72, 198-205.

36.

Stouffer, D.B. \& Bascompte, J. (2011). Compartmentalization increases food-web persistence. Proceedings of the National Academy of Sciences, 108, 3648-3652.

37.

Suweis, S., Bertuzzo, E., Mari, L., Rodriguez-Iturbe, L., Maritan, A. \& Rinaldo, A. (2012). On Species Persistence-Time Distributions. Journal of Theoretical Biology, 303, 15-24.

38.

Taylor, L.R. (1961). Aggregation, variance and the mean. Nature, 189, 732-735.

39.

Thompson, R.M., Brose, U., Dunne, J.A., Hall, R.O., Hladyz, S., Kitching, R.L., et al. (2012). Food webs: reconciling the structure and function of biodiversity. Trends in Ecology \& Evolution, 27, 689697.

40.

Vasseur, D.A. \& Gaedke, U. (2007). Spectral analysis unmasks synchronous and compensatory dynamics in plankton communities. Ecology, 88, 2058-2071.

41.

Widdicombe, C.E., Eloire, D., Harbour, D., Harris, R.P. \& Somerfield, P.J. (2010). Long-term phytoplankton community dynamics in the Western English Channel. Journal of plankton research, 32, 643-655. 

42.

Yee, T.W. (2010). The VGAM package for categorical data analysis. Journal of Statistical Software, 32, 1-34. \\ Page 21 of 26}

2

$267 \quad 1-34$


FIGURES

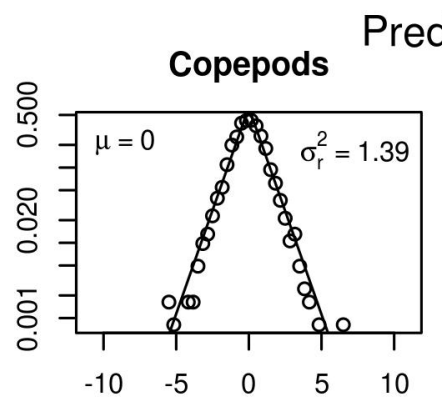

Omnivorous copepods

Consumers

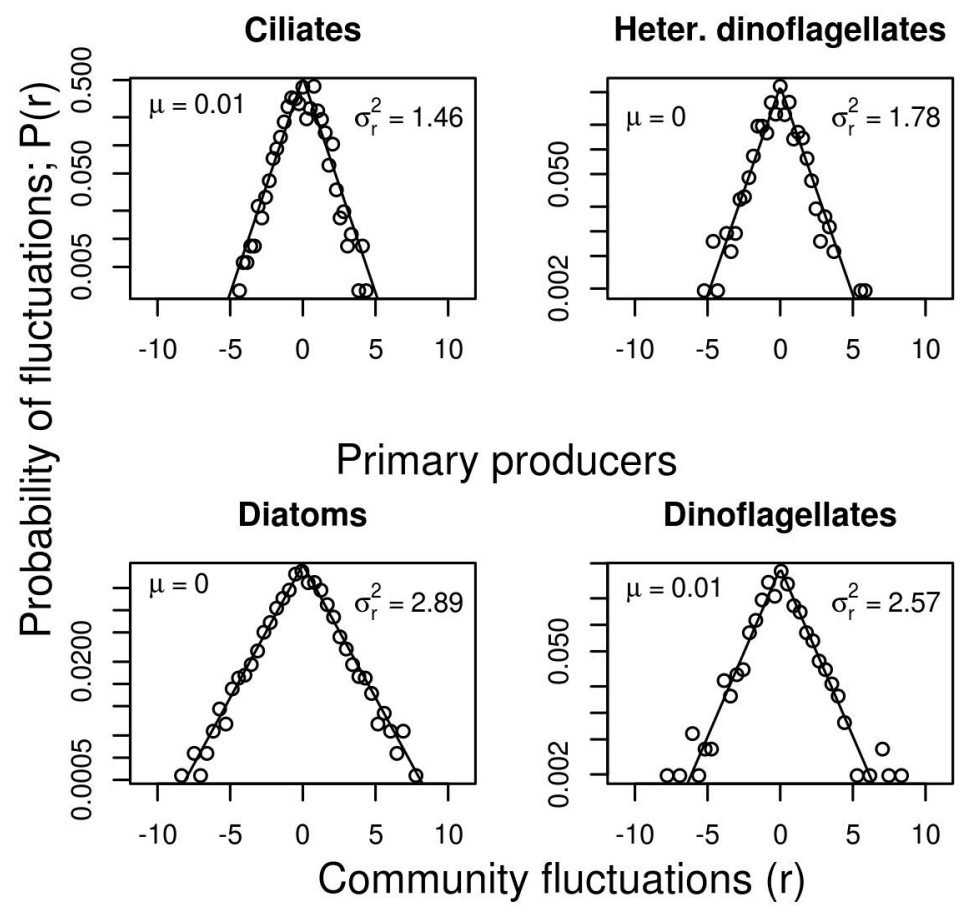

Figure 1.- Segura et al. 

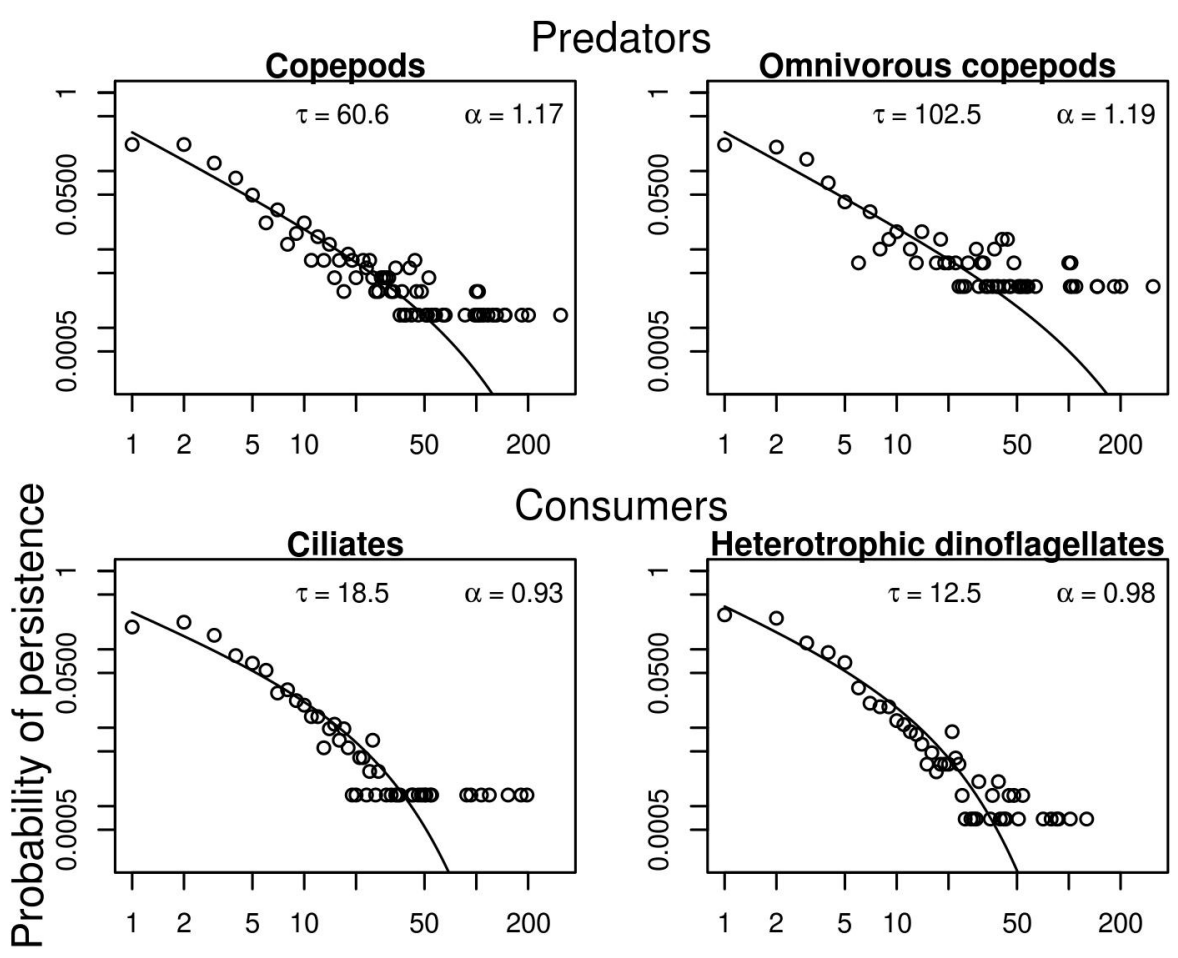

Primary producers

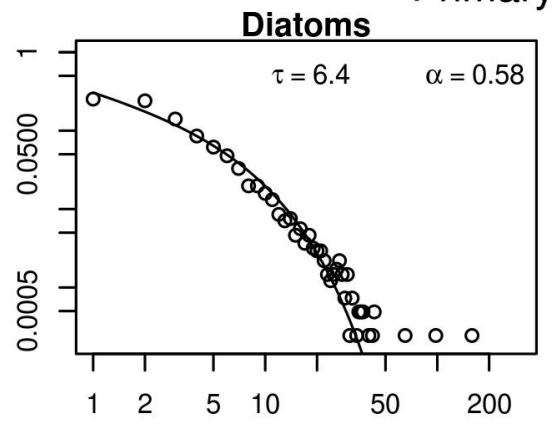

Dinoflagellates

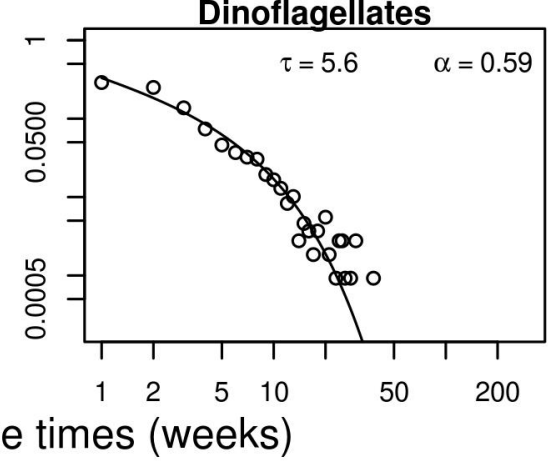

Figure 2.- Segura et al., 


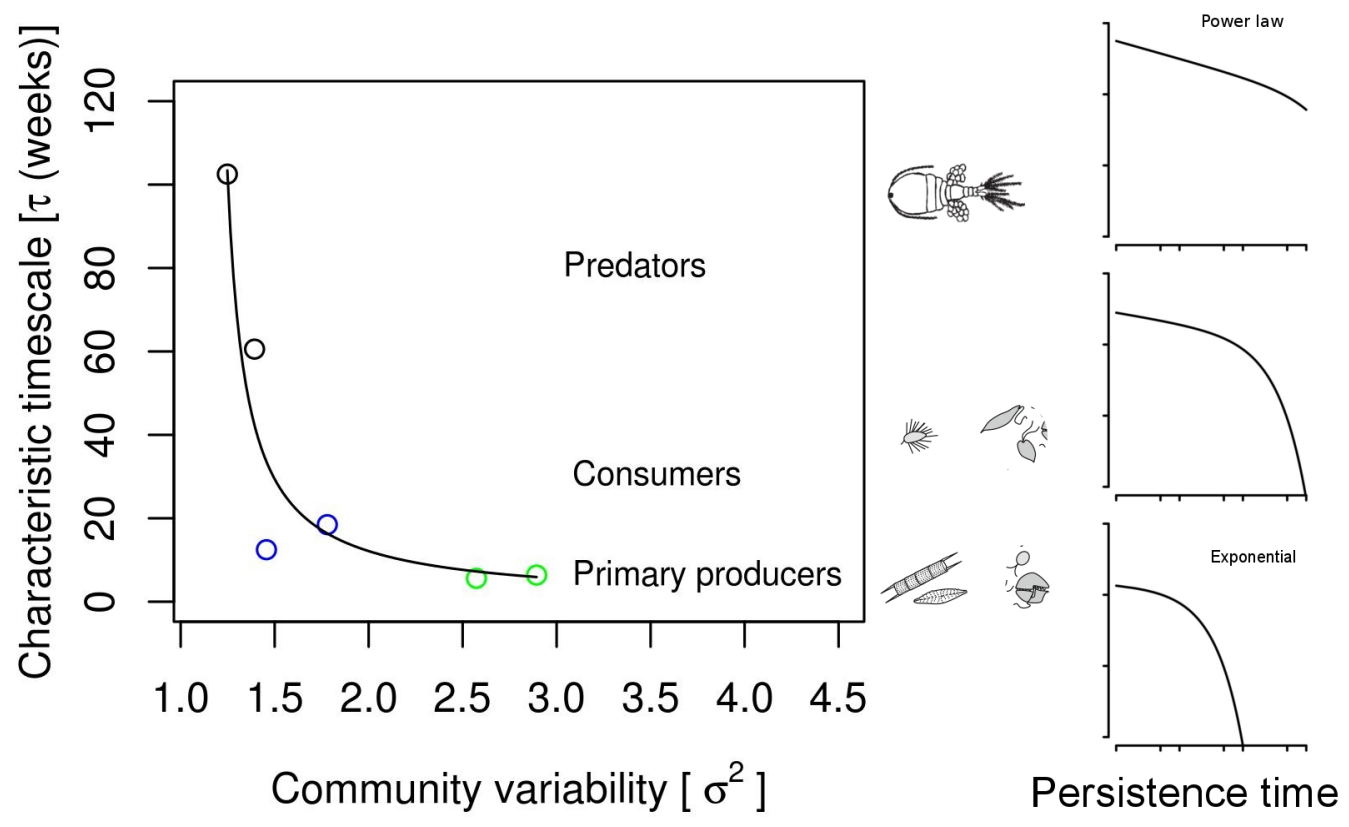

Figure 3.- Segura et al. 


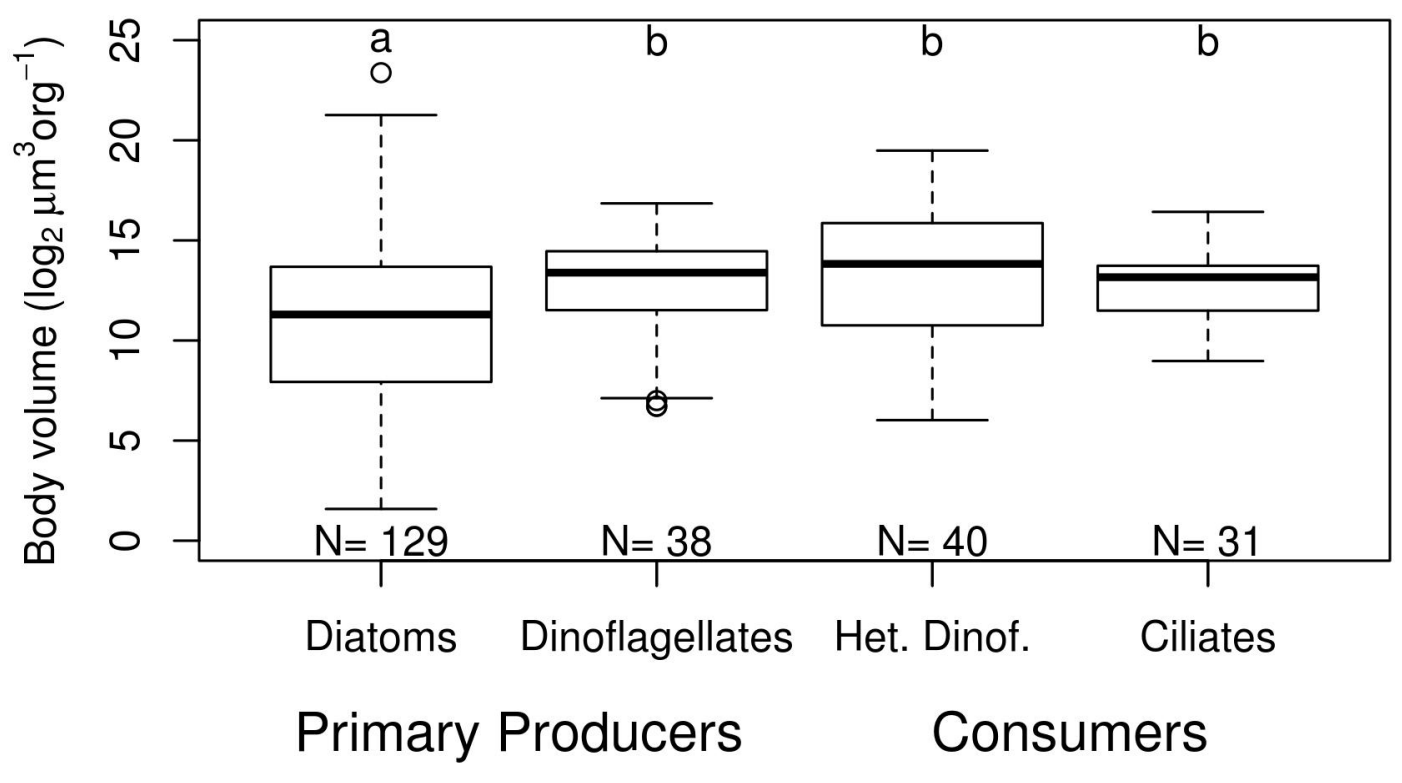

Figure 4.- Segura et al. 


\section{FIGURE LEGENDS}

282

283 Figure 1.- Aggregated population fluctuations for each functional group at Station L4 in the western 284 English Channel. Lines are the fitted models to original data and circles represent the middle point of 1

293 Figure 3.- Left, Negative relationship between variability and characteristic timescale times in the

294 Station L4 planktonic food web. The solid line is the best fit least squares non-linear regression $[\tau=10$ /

$\left.295\left(\sigma^{2}-1.14\right) ; \mathrm{N}=6\right]$. Right: schematic representation of the associated changes in the shape of the 296 persistence times distribution among trophic levels.

Figure 4.- Body size distributions of producers and consumers in the English Channel L4 Station. Same letter "b" represents no differences among groups according to a log likelihood ratio test. Diatoms presented different size structure according to a log-likelihood ratio test $(\mathrm{p}<0.05)$.

Figure 2.- Aggregated persistence time (APT) distribution for groups within each trophic level. The circles are the observed frequency distribution and the solid line is the fitted model $\left(\mathrm{P}(\mathrm{t})=\mathrm{C} \mathrm{t}^{-\alpha} \mathrm{e}^{-\mathrm{vt}}\right)$, where $\mathrm{C}$ is a normalization constant. Note the increase in the scaling exponent $(\alpha)$ and the characteristic timescale $(\tau=1 / v)$ from producers to predators. Lines are the models fitted to original data and circles represent the middle point of the histogram and was generated for visual purposes only. 